\title{
The Highs and Lows of FAIMS: Predictions and Future Trends for High Field Asymmetric Waveform Ion Mobility Spectrometry
}

Yuriy Zrodnikov and Cristina E. Davis*

Department of Mechanical and Aerospace Engineering, University of California, One Shields Avenue-Davis, CA 95616, USA

\section{Introduction}

High Field Asymmetric waveform Ion Mobility Spectrometry (FAIMS) [1], also known as Differential Mobility Spectrometry (DMS), has evolved as a useful method to separate ionized species in the gaseous phase [2]. All ions have a characteristic mobility value, which depends on the Electric field (E-field) and the surrounding gas mixture. The ion drift velocity is given by the product of the ion mobility and the E-field $(v=K E)$. For low electric fields below approximately 1500 $\mathrm{V} / \mathrm{cm}$ at atmospheric pressure [3], the mobility coefficient value does not change significantly as the E-field strength is varied. Traditional drift tube Ion Mobility Spectrometry (IMS) devices also operate in this regime. A sample is ionized and propelled through the drift tube using a gradient of electric fields. Only ions with a specific mobility value reach the detector(s) and are measured at corresponding drift times.

If the E-field is increased to the "high" strength regime above 1500 $\mathrm{V} / \mathrm{cm}$ at atmospheric pressure, the relationship between the ionic mobility coefficient and the ratio of electric field strength to gas number $(\mathrm{E} / \mathrm{N})$ becomes uniquely nonlinear for different ions. FAIMS, shown in Figure 1, takes advantage of these phenomena by using a rapidly time-varying electric field which varies from low to high strength [4]. Typical FAIMS devices feature a flow channel which includes two parallel planar or annular electrodes separated by a gap. The electric field is created by applying a waveform between the electrodes: one electrode is grounded while a high-frequency AC square waveform is applied to the other electrode. One cycle of the waveform induces an electric field which is initially high strength, and it is then switched to a low strength field in the opposite direction. The time period of each phase is specified such that the product of the electric field and the time period for which it is applied has a net value of zero over a single cycle. Gas-phase ionized sample species are swept through the drift tube by a constant carrier gas, while the E-field causes them to be displaced orthogonally to the gas flow through the device. Since an ion will have a different mobility in the high and low strength regimes, the ion will have a net displacement toward one of the electrodes over each cycle of the waveform. This displacement is counterbalanced by the application of a DC Compensation Voltage (CV). Each CV value allows ion(s) with that corresponding specific differential mobility to pass through the drift tube and reach the ion detector(s). The CV can also be specified to a value characteristic of a certain ion of interest to achieve an ion filtering regime, or it can be swept across a range to record abundance of all ion species that are present in a sample. Dispersion plots which show ion abundance for scans of $\mathrm{CV}$ at different values of the asymmetric waveform amplitude are commonly used to display sampled data. This usually provides extremely unique information for different chemicals, potentially leading to avenues for unique compound identification.

Frequently, FAIMS or DMS separation methods can be connected in series with other orthogonal analytical chemistry separation and detection techniques. For example, Gas Chromatography (GC) has been used to pre-separate complex analyte mixtures prior to FAIMS further separation and detection [5-8]. It has been shown that analytes of different categories such as aldehydes, ethers, esters, and alcohols have different retention times in the GC [5]. As components exit the GC column at a certain retention time, they enter the FAIMS drift tube where a range of compensation voltage is scanned. For each analyte, there will be a narrow band of CV values for which ions are able to traverse the drift tube and reach the detector. Ion abundance can be visualized as a $3 \mathrm{D}$ plot of compensation voltage and GC retention time. Abundance peaks are directly related to the concentration of a specific analyte in the sample. Multiple data analysis techniques $[6,7,9,10]$ have been used to identify ions and make distinctions by comparing the generated data with that of previously-obtained or calibrated results.

FAIMS and other atmospheric-pressure IMS techniques have also been successfully applied as a pre-filter for Mass Spectrometry (MS) systems [11-14]. An outlet at the end of the drift tube provides a path to the MS system, with pumps along the path to lower the pressure of the ion-carrying gas prior to injection. Ion funneling can be used to focus the flow into a narrow stream for the MS [15]. Cylindrical FAIMS systems offer the advantage of reduced ion loss at the FAIMS/ MS interface [16], however planar geometry with the FAIMS drift tube operating at atmospheric pressure has also been used [13].

\section{Applications}

Devices based on IMS have been implemented in many diverse

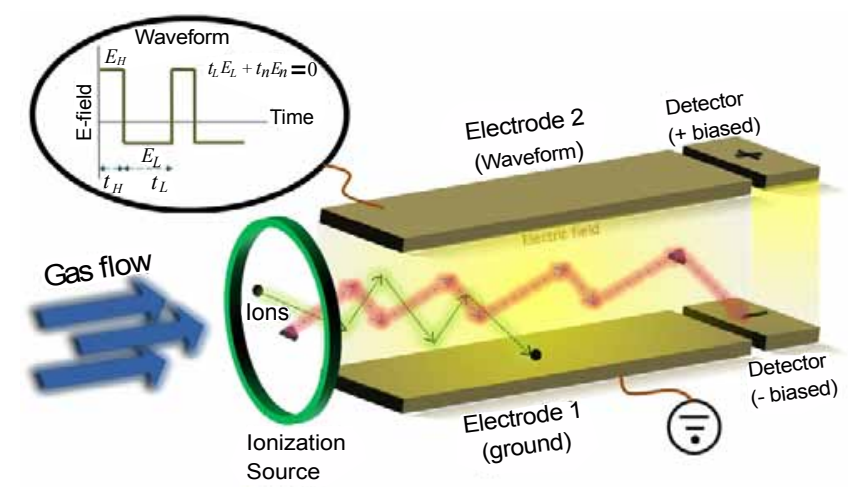

Figure 1: FAIMS overview.

*Corresponding author: Cristina E. Davis, Department of Mechanical and Aerospace Engineering, University of California, One Shields Avenue-Davis, CA 95616, United States, E-mail: cedavis@ucdavis.edu

Received May 17, 2012; Accepted May 17, 2012; Published May 19, 2012

Citation: Zrodnikov Y, Davis CE (2012) The Highs and Lows of FAIMS: Predictions and Future Trends for High Field Asymmetric Waveform lon Mobility Spectrometry. J Nanomed Nanotechol 3:e109. doi:10.4172/2157-7439.1000e109

Copyright: (c) 2012 Zrodnikov Y, et al. This is an open-access article distributed under the terms of the Creative Commons Attribution License, which permits unrestricted use, distribution, and reproduction in any medium, provided the original author and source are credited. 
applications. Traditionally IMS has been useful as a method for detecting explosive related compounds, and IMS devices are being widely employed in airports throughout the world [17]. Many explosive compounds form stable negatively-charged ions through ionization at atmospheric pressure, which IMS devices can effectively separate and detect. FAIMS detection of various explosive compounds is extremely specific and sensitive, with an example limit of detection as low as 80 parts-per-trillion (ppt) for 2,4,6-trinitrotoluene (TNT) [18]. In addition to detecting explosives, FAIMS devices have also been used for detecting chemical warfare agents [19] as well as narcotics [20].

Besides defense applications, a number of other studies using FAIMS technology have involved Volatile Organic Compound (VOC) detection and analysis [8]. Also, it has been demonstrated that it is possible to distinguish bacteria via biomarker VOCs produced during active proliferation [6]. Studies have also shown that nonvolatile metabolites in endospores can also be targeted for specific microorganism detection [10,21]. More recent biologically-related studies include health monitoring applications, in attempt to identify compounds in human Exhaled Breath Condensate (EBC) which then can be used to diagnose asthma or Chronic Obstructive Pulmonary Disease (COPD) [22,23]. Further, it was shown that GC/DMS can detect low concentrations of specific analytes even in the presence of a complex background mixture in human EBC [24].

Current GC/DMS research also includes identification of compounds from plants. This approach has been applied to analyze VOCs resulting from biogenic activity [25] by examining gas-phase VOCs in vivo from citrus trees [26]. There is also growing evidence that these technologies can be used for in-field detection of host-response to infection with specific pathogens of interest. The broad range of fields that have benefitted from IMS methods is a result of the technology's robustness. FAIMS in particular is relatively low-cost, and it can operate in atmospheric pressure using air or a specialty carrier gas. It also offers portability and real time sampling [27], and has the capability to be further scaled down for hand-held point detection systems.

\section{Limitations of Current Technologies}

Though IMS and FAIMS technologies have been applied successfully for variety of applications, the current methods still have some technical challenges that remain. One limitation has been difficulty to achieve reproducibility and cross-calibration between different instruments. This is primarily a result of imperfect square waveform generation, which is challenging to implement precisely in electronic systems. In general, RF waveform generators may have slight differences between units, and this can lead to small, but potentially significant, changes in FAIMS operation. Two different instruments may separate ions at slightly different compensation voltages. Each instrument can be independently calibrated, and for some applications this issue may not be significant (e.g. targeted analysis of specific compounds with very unique dispersion plots). However, for most emerging biological applications including metabolomics, this may affect each instrument's ability to collect and compare data seamlessly across many independent units. The RF waveform generation also draws a reasonably significant amount of power [28] and the waveform generator accounts for a large part of a typical FAIMS system's packaged size. Other parameters affecting FAIMS devices include the composition of the carrier gas mixture, flow rate [29], and ambient humidity. There is also evidence that certain device geometries may favor specific applications [16], and additional work on that front is likely to take place in upcoming years.

\section{Trends}

In the future it is likely that many of these limitations will be resolved. Because FAIMS is capable of operating at atmospheric pressure, it would be beneficial to develop a handheld and portable sensor that is completely portable as a full system. Progress in microfabrication and packaging allows further miniaturization of many system-level components, which also appears to be a promising way to improve overall performance. This may allow for adaptation of a FAIMS-like device for other emerging medical applications as well. Additionally, miniature systems require less power to achieve high strength fields due to closer gap spacing of the electrodes in the drift tube region [30]. It would also potentially allow more precise control over local electric fields, leading to higher sensitivity and lower limits of detection. With continuous progress, it is likely that fully handheld point detectors based on FAIMS will be achieved in the near future.

\section{Acknowledgements}

The authors would like to thank Dr. A. Aksenov for his substantial comments on the manuscript and discussions of FAIMS instrumentation platforms. This work was partially supported by several funding agencies. The content of this work is solely the responsibility of the authors and does not necessarily represent the official view of these agencies. Partial support is acknowledged from: UL1 RR024146 from the National Center for Research Resources (NCRR), a component of the National Institutes of Health (NIH), and NIH Roadmap for Medical Research; The Hartwell Foundation, Citrus Research Board, and the Florida Citrus Production Research Advisory Council (FCPRAC)

\section{References}

1. Buryakov IA, Krylov EV, Nazarov EG, Rasulev UK (1993) A new method of separation of multi-atomic ions by mobility at atmospheric-pressure using a high-frequency amplitude-asymmetric strong electric-field. Int J Mass Spectrom Ion Process 128: 143-148.

2. Eiceman GA, Karpas Z (2005) Ion mobility spectrometry, (2ndedn), Boca Raton: CRC Press.

3. Mason EA, McDaniel EW (1988) Transport properties of ions in gases. New York: Wiley.

4. Shvartsburg AA (2008) Differential ion mobility spectrometry: nonlinear ion transport and fundamentals of FAIMS. Boca Raton: CRC Press.

5. Eiceman GA, Tadjikov B, Krylov E, Nazarov EG, Miller RA, et al. (2001) Miniature radio-frequency mobility analyzer as a gas chromatographic detector for oxygen-containing volatile organic compounds, pheromones and other insect attractants. J Chromatogr A 917: 205-217.

6. Shnayderman M, Mansfield B, Yip P, Clark HA, Krebs MD, et al. (2005) Species-specific bacteria identification using differential mobility spectrometry and bioinformatics pattern recognition. Anal Chem 77: 5930-5937.

7. Zhao W, Sankaran S, Ibanez AM, Dandekar AM, Davis CE (2009) Two dimensional wavelet analysis based classification of gas chromatogram differential mobility spectrometry signals. Anal Chim Acta 647: 46-53.

8. Lambertus GR, Fix CS, Reidy SM, Miller RA, Wheeler D, et al. (2005) Silicon microfabricated column with microfabricated differential mobility spectrometer for GC analysis of volatile organic compounds. Anal Chem 77: 7563-7571

9. Eiceman GA, Wang M, Prasad S, Schmidt H, Tadjimukhamedov FK, et al. (2006) Pattern recognition analysis of differential mobility spectra with classification by chemical family. Anal Chim Acta 579: 1-10

10. Prasad S, Schmidt H, Lampen P, Wang M, Guth R, et al. (2006) Analysis of bacterial strains with pyrolysis-gas chromatography/differential mobility spectrometry. Analyst 131: 1216-1225.

11. Venne K, Bonneil E, Eng K, Thibault P (2005) Improvement in peptide detection for proteomics analyses using nanoLC-MS and high-field asymmetry waveform ion mobility mass spectrometry. Anal Chem 77: 2176-2186.

12. Handy R, Barnett DA, Purves RW, Horlick G, Guevremont R (2000) Determination of nanomolar levels of perchlorate in water by ESI-FAIMS-MS. Anal At Spectrom 15: 907-911. 
Citation: Zrodnikov Y, Davis CE (2012) The Highs and Lows of FAIMS: Predictions and Future Trends for High Field Asymmetric Waveform lon Mobility Spectrometry. J Nanomed Nanotechol 3:e109. doi:10.4172/2157-7439.1000e109

13. Levin DS, Vouros P Miller RA, Nazarov EG, Morris JC (2006) Characterization of gas-phase molecular interactions on differential mobility ion behavior utilizing an electrospray ionization-differential mobility-mass spectrometer system. Anal Chem 78: 96-106.

14. Steiner WE, Klopsch SJ, English WA, Clowers BH, Hill HH (2005) Detection of a chemical warfare agent simulant in various aerosol matrixes by ion mobility time-of-flight mass spectrometry. Anal Chem 77: 4792-4799.

15. Tang K, Li F, Shvartsburg AA, Strittmatter EF, Smith RD (2005) Twodimensional gas-phase separations coupled to mass Spectrometry for analysis of complex mixtures. Anal Chem 77: 6381-6388

16. Shvartsburg AA, Li F, Tang K, Smith RD (2006) High-resolution field asymmetric waveform ion mobility spectrometry using new planar geometry analyzers. Anal Chem 78: 3706-3714.

17. Ewing RG, Atkinson DA, Eiceman GA, Ewing GJ (2001) A critical review of ion mobility spectrometry for the detection of explosives and explosive related compounds. Talanta 54: 515-529.

18. Eiceman GA, Krylov EV, Krylova NS, Nazarov EG, Miller RA (2004) Separation of ions from explosives in differential mobility spectrometry by vapor-modified drift gas. Anal Chem 76: 4937-4944.

19. Kolakowski BM, Mester Z (2007) Review of applications of high-field asymmetric waveform ion mobility spectrometry (FAIMS) and differential mobility spectrometry (DMS). Analyst 132: 842-864.

20. Pollard MJ, Hilton CK, Li H, Kaplan K, Yost RA, et al. (2011) lon mobility spectrometer-field asymmetric ion mobility spectrometer-mass spectrometry. Int J Ion Mobil Spectrom 14: 15-22.

21. Krebs MD, Zapata AM, Nazarov EG, Miller RA, Costa IS, et al. (2005) Detection of biological and chemical agents using differential mobility spectrometry (DMS) technology. IEEE Sens J 5: 696-703.
22. Schivo M, Zhao W, Aksenov A, Kenyon N, Davis C (2011) Metabolomic Analysis of Exhaled Breath Condensates (EBC) In Asthma And COPD. J Investig Med 59: 205-205.

23. Basanta M, Jarvis RM, Xu Y, Blackburn G, Tal-Singer R, et al. (2010) Noninvasive metabolomic analysis of breath using differential mobility spectrometry in patients with chronic obstructive pulmonary disease and healthy smokers. Analyst 135: 315-320.

24. Davis CE, Bogan MJ, Sankaran S, Molina MA, Loyola BR, et al. (2010) Analysis of Volatile and Non-Volatile Biomarkers in Human Breath Using Differentia Mobility Spectrometry (DMS). IEEE Sens J 10: 114-122.

25. Dandekar AM, Martinelli F, Davis CE, Bhushan A, Zhao W, et al. (2010) Analysis of Early Host Responses for Asymptomatic Disease Detection and Management of Specialty Crops. Crit Rev Immunol 30: 277-289.

26. Aksenov A, WHK C, Zhao W, Bardaweel H. Martinelli F, et al. (2012) Reagentless Detection of Citrus Pathogens Using Differential Mobility Spectrometry. Citrograph 3: 54-56.

27. Manard MJ, Trainham R, Weeks S, Coy SL, Krylov EV, et al. (2010) Differentia mobility spectrometry/mass spectrometry: The design of a new mass spectrometer for real-time chemical analysis in the field. Int J Mass Spectrom 295: 138-144.

28. Papanastasiou D, Wollnik H, Rico G, Tadjimukhamedov F, Mueller W, et al. (2008) Differential mobility separation of ions using a rectangular asymmetric waveform. J Phys Chem A 112: 3638-3645.

29. Barnett DA, Ells B, Guevremont R (2000) Evaluation of carrier gases for use in high-field asymmetric waveform ion mobility spectrometry. J Am Soc Mass Spectrom 11: 1125-1133.

30. Shvartsburg AA, Smith RD, Wilks A, Koehl A, Ruiz-Alonso D, et al. (2009) Ultrafast Differential Ion Mobility Spectrometry at Extreme Electric Fields in Multichannel Microchips. Anal Chem 81: 6489-6495. 